\title{
Excitation of guided-mode resonances in thin film silicon solar cells
}

\author{
F.-J. Haug, K. Söderström, A. Naqavi, C. Ballif \\ Ecole Polytechnique Fédérale de Lausanne (EPFL), Institute of Microengineering (IMT), \\ Photovoltaics and Thin Film Electronics Laboratory, \\ Rue A.-L. Breguet 2, CH-2000 Neuchâtel, Switzerland
}

\begin{abstract}
Thin film silicon solar cells are attractive for photovoltaics; however, the poor charge transport in this material requires that the devices are thinner than the absorption length. Adequate absorption can nevertheless be achieved by light scattering at textured interfaces because light can get trapped inside the absorber layer if it is scattered into angles above the critical angle of total internal reflection. This situation can be identified with the propagation of a guided mode in a waveguide where silicon plays the role of the high index guiding medium and the interface texture serves to couple the incident light to modes via grating coupling. We present an experimental realization of a solar cell structure on a line grating where the enhanced photocurrent can be clearly related to resonant excitation of waveguide modes.
\end{abstract}

\section{INTRODUCTION}

Thin film silicon is an interesting option for low cost solar energy production because its manufacturing technology is well mastered and it is an abundant material. Its most fundamental limitation against large scale production is related to its poor electronic quality compared to its crystalline counterpart. Poor charge transport poses several fundamental limitations on the device design. For example, the absorber layer thickness is normally much smaller than the absorption length, particularly for light with energy close to the band gap [1]; therefore, state-of-the-art devices require additional elements that enhance the absorption within the absorber layer. The earliest, and so far experimentally most successful approach employs light scattering at textured interfaces [2]. Light that is scattered beyond the critical angle for total internal reflection is thus trapped into the absorber film. Obviously, the condition of total internal reflection is not perfect in the presence of interface texture; light trapping is therefore an equilibrium between in-coupling which results in the desired absorption enhancement in the silicon film, and the undesired out-coupling.

In the terminology of the optics community, the silicon absorber layer can be identified with the high index guiding medium, the metal film at the back that serves as reflector and electric contact translates to the cladding, and the interface texture establishes the grating coupling between the external radiation field and eigen-modes of the multilayer stack. Different from standard waveguides, typical dimensions of a solar cell are likely to support multiple modes, the guiding medium is absorbing and the requirement of broad-band coupling means a spectral range between 600 and 750 $\mathrm{nm}$ for amorphous solar cells and an even larger range from 700 to $1000 \mathrm{~nm}$ for microcrystalline solar cells.

In this contribution, we describe an amorphous solar cell as planar waveguide and discuss the main features of the dispersion relation, using realistic thicknesses for all component films and including the dispersion of the respective refractive indices. The theoretical simplification of flat interfaces is checked by measuring resonant absorption enhancement in the external quantum efficiency of a solar cell on a line grating. Thanks to the one-dimensionality of the grating, polarization phenomena are distinguished. 


\section{EXPERIMENTAL}

We used a commercially available grating with a period of $560 \mathrm{~nm}$ and blazed shape (Thorlabs, GR18). In order to be compatible with the subsequent fabrication steps, the grating was replicated on glass substrates, using a UV-curable lacquer [3]. The cross section in Figure 1 shows a peak-to-valley depth of $140 \mathrm{~nm}$ and a saw-tooth with slightly rounded tip. A. The grating was covered with a back reflector consisting of a sputtered double layer of silver $(120 \mathrm{~nm})$ and zinc-oxide $(60 \mathrm{~nm})$. The silicon film is grown in an n-i-p sequence with a total thickness of $200 \mathrm{~nm}$ and the front contact consists of ITO $\left(\mathrm{In}_{2} \mathrm{O}_{3}-\mathrm{SnO}_{2}\right)$ with a thickness of $65 \mathrm{~nm}$ which serves as anti-reflection coating [4].

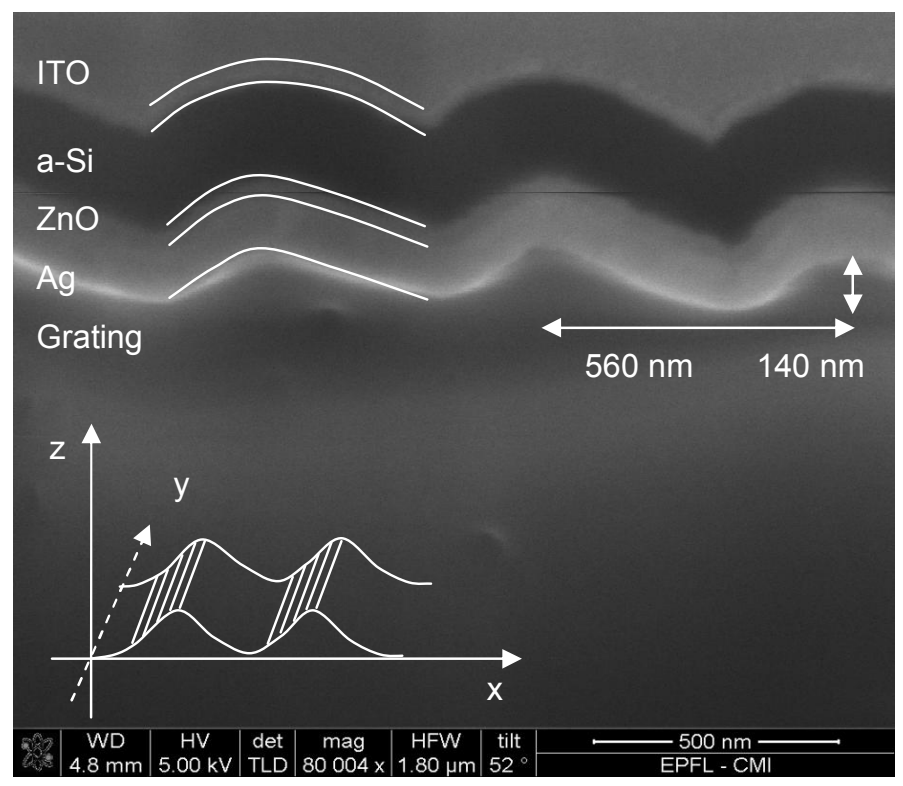

Figure 1: Cross section of the solar cell on the grating substrate. The inset illustrates the orientation of the coordinates.

The polarization resolved external quantum efficiency (EQE) was measured under perpendicular incidence with a broad-band wire polarizer (ProFlux, Moxtek). Figure 1 illustrates the coordinate system; in s-polarization, the E-field of is parallel to the y-axis, in p-polarization the E-field is contained in the $\mathrm{x}-\mathrm{z}$ plane. The EQE is the probability of creating and collecting an electron-hole pair per incident photon at a specific wavelength in the illumination spectrum.

\section{THEORY}

Eigen-modes of a multilayer system are easily found with the pole method [5]. Based on the assumption that a resonance corresponds to a singularity of the system, the pole method simply looks for zeros in the denominator of the Fresnel coefficient. Fresnel coefficients of multilayer stacks are defined recursively, starting from the coefficients for the interface between two component media $i$ and $j$. For s- and p-polarization they are defined as follwos:

$$
r_{i j}^{s}=\frac{S_{j}-S_{i}}{S_{j}+S_{i}} \text { and } r_{i j}^{p}=\frac{\varepsilon_{i} S_{j}-\varepsilon_{j} S_{i}}{\varepsilon_{i} S_{j}+\varepsilon_{j} S_{i}}
$$

The first and the last layers of the stack assumed to be semi-infinite, the propagation constants $S_{i}$ are defined for every individual layer $i$ in terms of the complex dielectric constant $\varepsilon_{\mathrm{i}}$ and $k_{0}=2 \pi / \lambda$, the modulus of the wave vector in air:

$$
S_{i}^{2}=k_{0}^{2}-\varepsilon_{i} k_{\|}^{2}
$$

For more than two layers, Fresnel coefficients are defined via the single interface coefficients of eq. (1), using coherent propagation across the film thicknesses $d_{i}$ : 


$$
r_{1 \ldots n}=\frac{r_{12} \cdot r_{2 \ldots n} \cdot \exp \left\{-2 S_{2} d_{2}\right\}}{r_{12}+r_{2 \ldots n} \cdot \exp \left\{-2 S_{2} d_{2}\right\}}
$$

Eqns. (1) to (3) were used to determine the dispersion relation for a solar cell stack using five layers [6]; the silver layer is assumed semi-infinite and we considered a $60 \mathrm{~nm}$ thick $\mathrm{ZnO}$ buffer layer, a $200 \mathrm{~nm}$ thick amorphous silicon film, a $65 \mathrm{~nm}$ thick ITO front contact and an air space above.

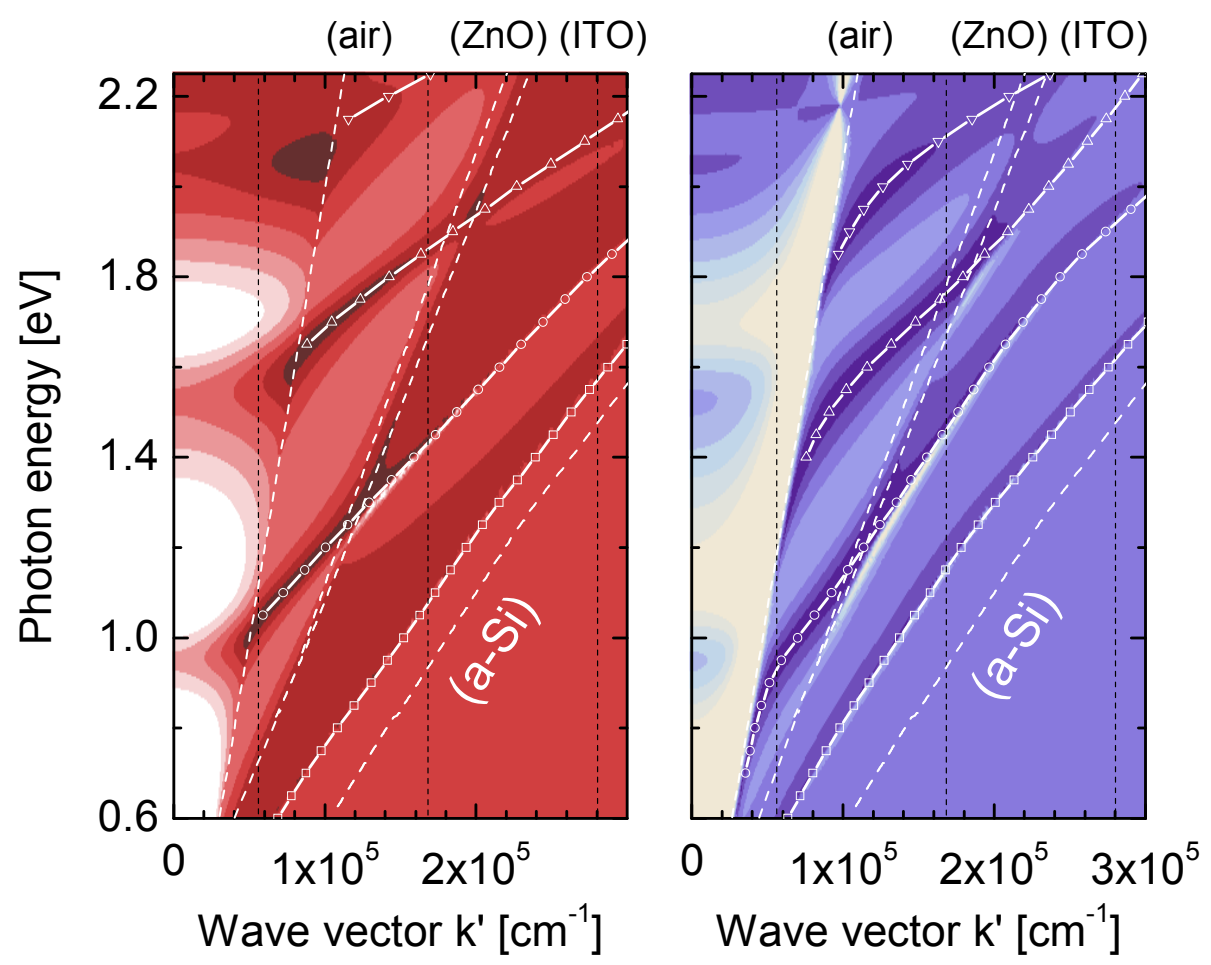

Figure 2: Dispersion diagrams in s-(left) and p-polarization (right). The symbols denote the individual modes of the solar cell stack, the dashed lines represent light lines of the respective materials. Vertical lines denote the Brillouin zones for a grating with period of $560 \mathrm{~nm}$. The background is a contour-plot of $\operatorname{Abs}\left(\mathrm{D}\left(\boldsymbol{r}_{1 \ldots 5}\right)\right)^{2}$.

Before discussing the dispersion relation of the solar cell stack, we would like to point out a few general aspects of dispersion diagrams like the one shown in Figure 2. Propagation of light in an unbounded medium corresponds to straight lines according to the dispersion relation of photons which is given by $\omega(k)=(c k) / n$. In Figure 2, the lines are not exactly straight because of the refractive index dispersion. Guided modes can be defined approximately by requiring nodes at the boundaries of the guiding medium; in case of the fundamental or zero-order mode, half of the wavelength can be fit into the thickness of the high-index layer. Likewise, higher order modes can be defined by fitting integer multiples. This definition explains the cut-off condition because modes with wavelengths larger than twice the guiding film thickness cannot be guided.

Figure 2 shows the guided mode structure of the solar cell stack. The left and right panels correspond to s- and p-polarization, respectively, and their backgrounds show contour-plots of the squared modulus of the denominator of $r_{1} \ldots 5$. Dark shades correspond to values close to zero. The symbols represent numeric solutions. The cut-off condition of the zero-order mode (squares) is not observed, but with increasing energy first order (circles), second order (up-triangles) and third order (down-triangles) modes appear alternatingly in the two polarization directions. At their onset, all modes lie close to the light line of air. This observation is explained by the dielectric nature of the ITO front contact; compared to the silver back contact, the confinement at the front of the device is relatively weak. At their onset, the modes resemble standing waves in z-direction rather than guided modes in x-direction. With increasing energy, all modes approach the light line of amorphous silicon.

The region towards the left of the air-line represents a continuum of radiation modes. The radiation modes are straight lines that emerge from the origin, their slope is related to their angle of 
incidence with respect to the average cell surface. The shown light line of air represents grazing incidence, the light line of perpendicular incidence coincides with the vertical energy axis. Note that none of the continuum modes intersects with the guided modes, conservation of energy and momentum forbids an interaction of radiation modes with Eigen-modes. Darker shades in the contourplot reveal nevertheless that a certain amount of resonant behaviour does exist in this region because of multiple reflections into angles below the critical angle.

Interactions between the radiation modes and guided modes can be established by grating coupling. In the presence of a periodic interface texture, every light line that emerges from the origin can pick up multiples of the reciprocal lattice vector that translates it into the centre of higher order Brillouin zones. Note that this approach also applies to random interface textures by means of their spatial Fourier expansion. For the case of sinusoidal textures, relations for the strength of out-coupling from a guided mode to the radiation field have been worked out in ref. [7]. In a future contribution, we plan to adapt the theory to the inverse situation of in-coupling from the radiation field to a (lossy) guided mode. In the remainder of this contribution, we would like to present experimental evidence on grating coupling in a real solar cell device.

\section{ABSORPTION ENHANCEMENT IN SOLAR CELLS BY GRATING COUPLING}

The excitation of guided modes by grating coupling was investigated by manufacturing a complete solar cell on a grating with period of $560 \mathrm{~nm}$. Figure 3 shows the polarization resolved EQE of the weakly absorbing region; in addition to the Fabri-Perot interferences that are also observed on flat cells, the external quantum efficiency shows clearly resolved resonances [8]. The energies of the resonances are transferred to Figure 4 which shows the weakly absorbing region of Figure 2. Positions of the resonances are tentatively assigned by arrows; in s-polarization, the coupling at $1.63 \mathrm{eV}$ is most likely to the $\mathrm{s} 1$ mode in the third Brillouin zone, the resonance at $1.81 \mathrm{eV}$ can be attributed to the $\mathrm{s} 0$ mode in the fourth Brillouin zone. In p-polarization, coupling at $1.91 \mathrm{eV}$ involves the $\mathrm{p} 2$ mode in the third Brillouin zone, whereas the coupling at $1.79 \mathrm{eV}$ is likely to be with the p0 mode in the fourth Brillouin zone.
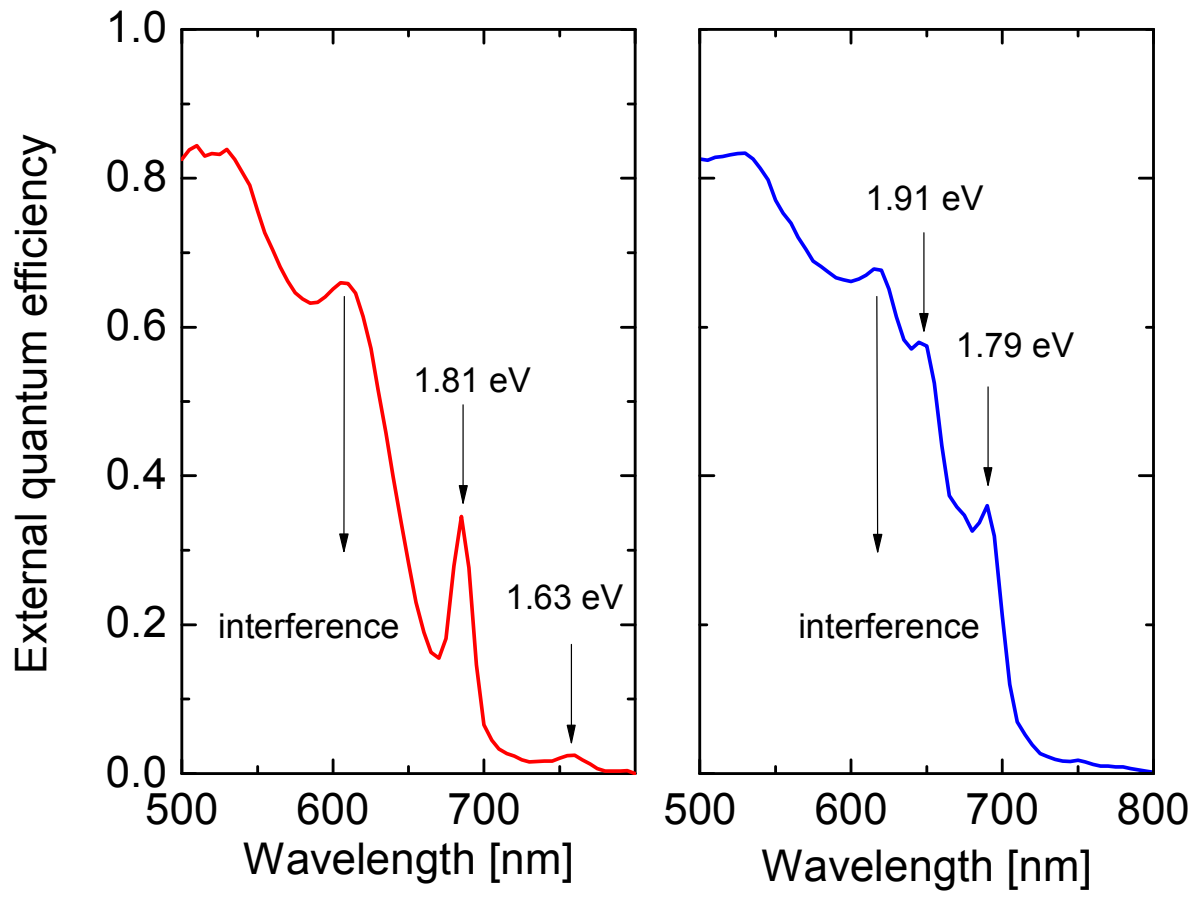

Figure 3: Polarization resolved external quantum efficiency (EQE) of a solar cell on a line grating with a period of $560 \mathrm{~nm}$ in s-(left) and p-polarization (right). 
We note that the energies of the dispersion diagram of Figure 4 would suggest that the scoupling in the fourth Brillouin zone occurs at lower energy than the p-coupling. This discrepancy can possibly be explained by the fact that the theoretical dispersion diagram was determined on the basis of flat interfaces but did not take into account the true nature of the interface texture.

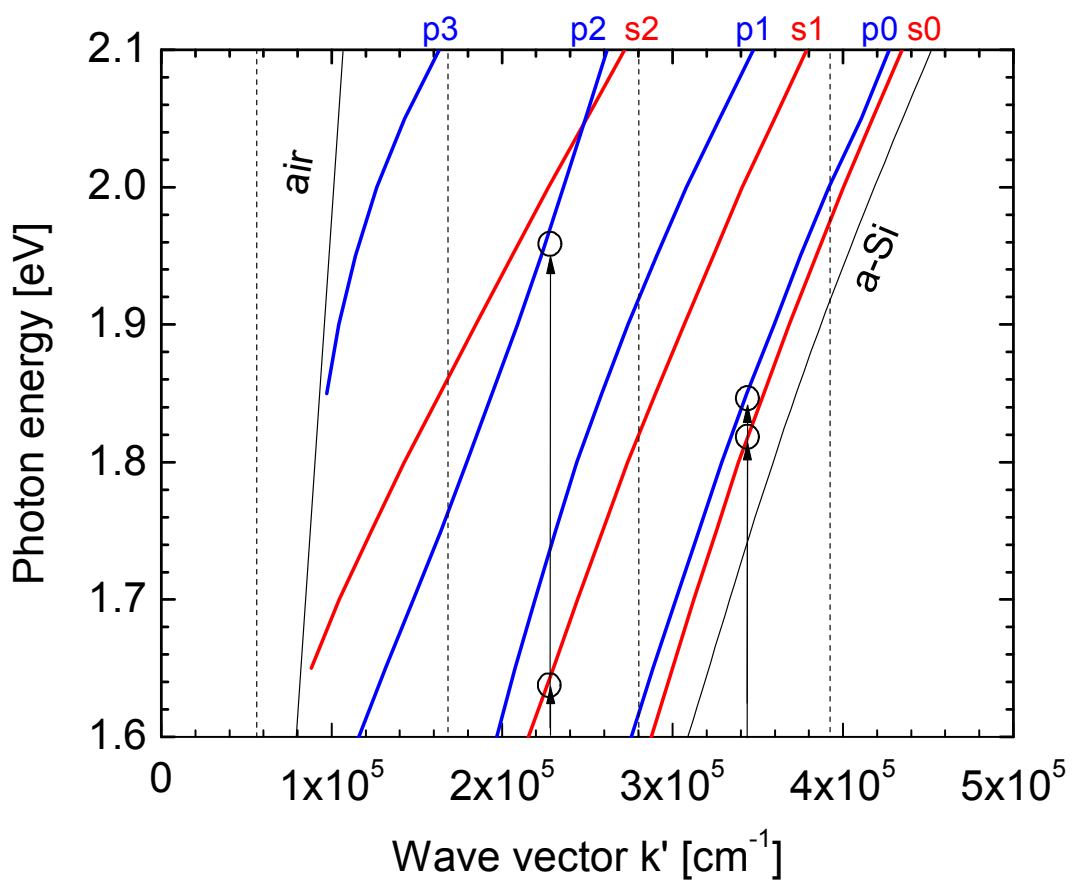

Figure 4: Detail of the dispersion diagram of Figure 2, only the weakly absorbing region is shown; grating coupling for the four resonances of Figure 3 is indicated tentatively.

\section{FIELD DISTRIBUTION}

In order to assess the amount of absorption enhancement, the amplitude of the electromagnetic field throughout the structure must be known. Figure 5 shows the s-polarized field intensity for the wavelength of the dominant resonance at $690 \mathrm{~nm}$ calculated with rigorous coupled wave analysis (RCWA) for the exact interface texture shown in Figure 1 [9]. For the shown 1D problem on the line grating, the required computing power is still reasonable. However, for more complicated scenarios it would be desirable to revert to analytic approximations, provided they can provide predictive power.
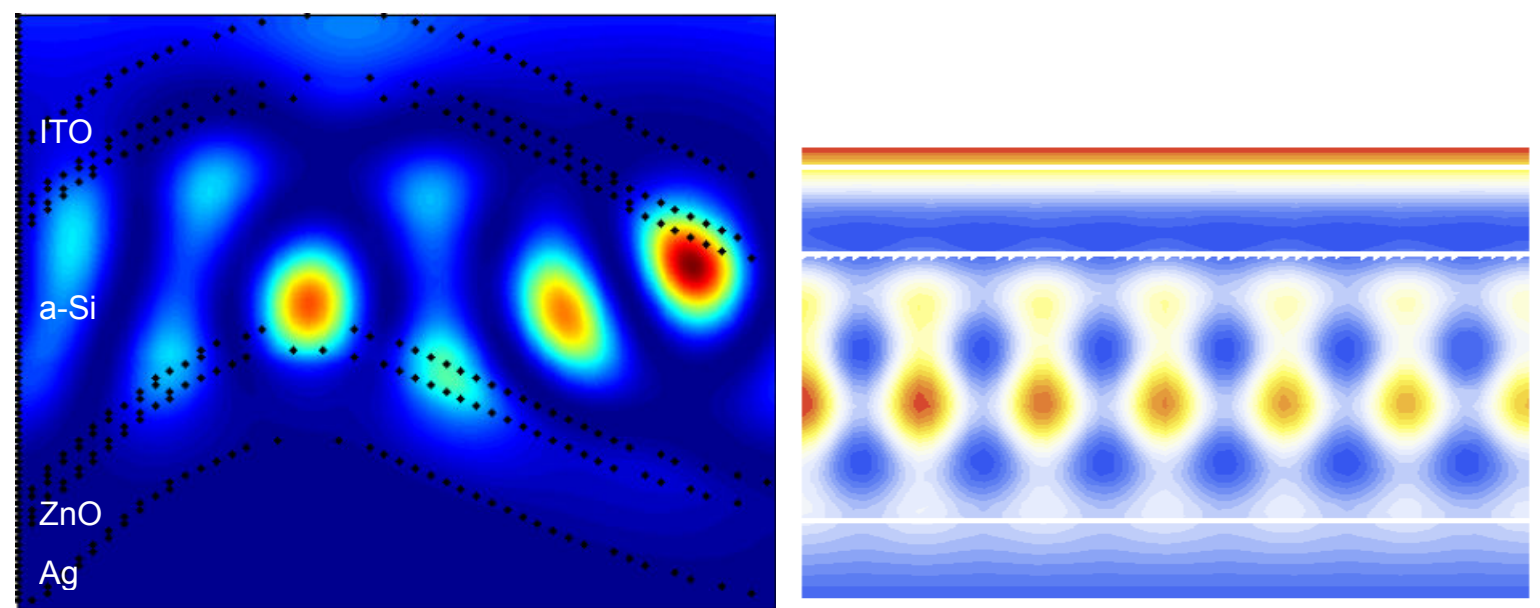

Figure 5: Intensity of the electric field in s-polarization at the resonance wavelength of $690 \mathrm{~nm}$. The left panel shows the rigorous solution, the right panel illustrates the flat waveguide approximation. The horizontal scale corresponds to one period of $560 \mathrm{~nm}$. 
The rather complicated field profile of the exact solution in Figure 5 can be understood by superimposing the field intensity of the $\mathrm{s} 0$ guided at $k_{\|}=3.43 \times 10^{5} \mathrm{~cm}^{-1}$ with the interference pattern of the Fabry-Perot resonance. The former shows 6 clearly resolved maxima in x-direction because this value of the wave vector accommodates three full wavelengths within the grating period, and one single maximum in z-direction because the order number zero means that there are no nodes within the guiding film. The interference pattern adds three maxima in the z-direction; the resulting superposition of the fields yields a surprisingly accurate correspondence with the exact calculation.

\section{CONCLUSIONS}

We investigated the effect of a periodic interface texture on the absorption enhancement in amorphous silicon solar cells. In the experimental part, devices with periodic interfaces were found to exhibit clearly resolved absorption signatures in the weakly absorbing region. In the theoretical part, we treated the solar cell in terms of a planar waveguide that supports multiple modes. With the help of the dispersion diagram, we were able to explain the absorption enhancement by the resonant excitation of waveguide modes and to attribute the resonances to specific mode orders. Having identified the modes that participate in a resonance, their coupling strength can be determined with existing theories; ultimately this should lead to a parameter or a few parameters that can be tuned for better absorption enhancement.

\section{ACKNOWLEDGEMENTS}

We thankfully acknowledge Dr Aïcha Hessler and Caroline Calderone for FIB cross sectioning and SEM imaging. This work was funded by the European Union within the project Si-Light (Contract No 241277) and by the Swiss National Science Foundation (Contract No. 2000021_125177/1).

\section{REFERENCES}

1. D. E. Carlson and C. R. Wronski, "Amorphous Si Solar Cell" Applied Physics Letters 28(11), p. 671-673 (1976)

2. H. W. Deckman, C. R. Wronski, H. Witzke, and E. Yablonovitch, "Optically enhanced amorphous silicon solar cells" Applied Physics Letters 42(11), p. 968-970 (1983)

3. K. Söderström, J. Escarré, O. Cubero, F.-J. Haug, S. Perregaux, and C. Ballif, "UV nano imprint lithography technique for the replication of back reflectors for n-i-p thin film silicon solar cells" Progress in Photovoltaics: Research and Applications 19(2), p. 202-210 (2011)

4. H. Okamoto, Y. Nitta, T. Adachi, and Y. Hamakawa, "Glow discharge produced amorphous silicon solar cells" Surface Science 86, p. 486-491 (1979)

5. S. Shakir and A. Turner, "Method of poles for multilayer thin-film waveguides" Applied Physics A: Materials Science \& Processing 29(3), p. 151-155 (1982)

6. F.-J. Haug, K. Söderström, A. Naqavi, and C. Ballif, "Resonances and absorption enhancement in thin film silicon solar cells" accepted for application in Journal of Applied Physics (2011)

7. I. Avrutsky, A. Svakhin, and V. Sychugov, "Interference phenomena in waveguides with two corrugated boundaries" Journal of Modern Optics 36(10), p. 1303-1320 (1989)

8. K. Söderström, F. Haug, J. Escarré, O. Cubero, and C. Ballif, "Photocurrent increase in n-ipthin film silicon solar cells by guided mode excitation via grating coupler" Applied Physics Letters 96, p. 213508 (2010)

9. A. Naqavi, K. Söderström, F. J. Haug, V. Paeder, T. Scharf, H. P. Herzig, and C. Ballif, "Understanding of photocurrent enhancement in real thin film solar cells: towards optimal onedimensional gratings" Optics Express 19(1), p. 128-140 (2011) 